\title{
LA COMPETITIVIDAD INTERNACIONAL DE LA INDUSTRIA ALGODONERA ESPAÑOLA (1830-1860)
}

\author{
JOAN R. ROSÉS * \\ Universidad Carlos III de Madrid
}

\begin{abstract}
RESUMEN
Con el fin de explicar las grandes diferencias de precios entre los tejidos de algodón británicos y españoles, este artículo se ocupa de medir el coste de las materias primas y estimar los niveles de productividad total de los factores (PTF) en ambos países. Ambos cálculos sugieren una relación directa entre la falta de competitividad internacional de la industria española y los altos niveles de protección. Así, a lo largo del artículo se demuestra que la especialización inadecuada, que fue una consecuencia directa de los altos aranceles, redujo los niveles de eficiencia en España porque las fábricas locales producían bienes demasiado sofisticados para las habilidades de su fuerza de trabajo.
\end{abstract}

\section{ABSTRACT}

To explain the large differences in prices between Spanish and British cotton goods I measure the relative cost of raw materials and estimate relative TFP levels. Both calculations lead to a relation between Spain's poor performance in international markets and high levels of protection. Thus, I find that inadequate specialization, which was consequence of high tariffs, reduced TFP levels in Spain because local factories produced goods too sophisticated for the abilities of their workforce.

* Una versión previa de este trabajo fue presentada en el seminario organizado por la Universidad Menéndez Pelayo en Cuenca y en la Universidad Carlos III de Madrid. Debo agradecer las críticas y comentarios, entre otros, de Juan Carmona, Mar Cebrián, Carles Sudrià y dos evaluadores anónimos. Obviamente, todos los errores y omisiones del texto son de mi absoluta responsabilidad. 


\section{INTRODUCCIÓN}

La industria algodonera fue una de las principales protagonistas de las primeras fases de la industrialización española ${ }^{1}$. Así, de 1830 a 1860 la producción nacional de tejidos se multiplicó casi por ocho ${ }^{2}$. Pese a este notable éxito en términos de fabricación la industria algodonera española fracasó estrepitosamente en los mercados internacionales consiguiendo exportar sólo una minúscula parte de sus productos e incluso tuvo problemas, pese a los elevados niveles de protección, para copar el mercado interior de géneros de algodón ${ }^{3}$. A partir de esta evidencia, no resulta difícil concluir que nos encontramos ante un caso flagrante de industria no competitiva.

Se podrían avanzar diversas hipótesis para explicar esta falta de competitividad internacional de la industria algodonera española. Cualquier economista nos remitiría, como primera explicación plausible, a la teoría de la ventaja comparativa. Según el modelo de Heckscher-Ohlin, las exportaciones y las importaciones de un país reflejan la abundancia relativa o escasez de los factores de producción en ese país ${ }^{4}$. Por tanto, se podría decir que España no era competitiva en la fabricación de géneros de algodón debido, simplemente, al alto coste de oportunidad de los factores de producción que intervenían en la producción de esa clase de tejidos. Esta explicación, que tiene notables visos de credibilidad, parece difícil de aceptar si consideramos que Italia, un país con una dotación de factores y recursos naturales parecida a la española, consiguió una notable cuota del mercado internacional de tejidos de algodón ${ }^{5}$.

Otra explicación de esta falta de competitividad sería la presencia de alguna clase de «desventaja natural» que impediría a los industriales espa-

${ }^{1}$ Por razones de espacio, en el texto que sigue se excluye una historia detallada de la evolución de la industria algodonera en España. Para aquellos lectores que deseen más información les remito a Nadal (1974) y (1991), Maluquer (1985) y Thomson (1994). Por la misma razón tampoco se incluye un resumen del debate sobre las consecuencias de la protección; al respecto puede verse, entre otros, Tena (1999), Tena y Tirado (1996), Serrano Sanz (1997) y Sabaté (1996).

${ }^{2}$ Nadal (1974).

${ }^{3}$ Sudrià (1983), Prados de la Escosura (1984). La industria algodonera española estaba protegida desde 1802 con la prohibición de la importación de géneros extranjeros de algodón, Nadal (1974). En la década de 1840 , el alcance de la prohibición se redujo, ya que se permitió la importación de géneros de algodón fabricados con hilos superiores al número 60 . Ronquillo (1851-1857). Sin embargo, esta modificación de la estructura del arancel no afectó a la industria española que no producía géneros tan finos. En consecuencia, durante todo el período que vamos a estudiar, la protección de la industria algodonera fue extremadamente importante.

${ }^{4}$ Flam y Flanders (1991).

5 Zamagni (1993). 
ñoles ser competitivos internacionalmente. Por ejemplo, el alto coste del carbón impediría a las industrias españolas competir con las extranjeras ${ }^{6}$. Este argumento, sin embargo, no se corresponde demasiado bien con la experiencia de la industria algodonera, ya que el combustible representaba una porción pequeña de los costes de producción, el carbón podía ser reemplazado fácilmente por la energía hidráulica, e Italia, con restricciones energéticas similares a las españolas, fue capaz de producir competitivamente ${ }^{7}$.

Asimismo podríamos pensar que la industria algodonera española no era competitiva en los mercados internacionales por su retraso tecnológico respecto a otros países. Este retraso tecnológico podría explicarse de varias maneras. Se podría sugerir que los empresarios españoles fueron incapaces de adoptar las tecnologías foráneas. Es decir, que los empresarios españoles se mostraban adversos al riesgo y tenían una actitud refractaria hacia las innovaciones ${ }^{8}$. También, se podría argüir que la industria algodonera española se encontraba en su fase de aprendizaje de las nuevas tecnologías y que por ello no era capaz de emular a sus competidoras de los países más avanzados ${ }^{9}$. Ninguna de estas hipótesis, empero, cuadra con la experiencia de la industria algodonera. Si bien es cierto que la industria española se mecanizó con un cierto retraso respecto a la industria británica o la belga, a partir de finales de la década de 1830, tampoco es menos cierto que lo hizo con inusitada rapidez. Así, en 1860, las empresas algodoneras españolas producían sus tejidos con la misma tecnología y métodos que sus homólogas británicas utilizando como principal combustible el carbón. En otras palabras, la fase de «industria naciente» de la industria algodonera española debió acabar en la década de 1850 y, a partir de entonces, utilizó siempre la misma tecnología que las industrias algodoneras más avanzadas ${ }^{10}$.

Otra de las presuntas razones de la falta de competitividad de la industria española podría ser que el mercado internacional de los géneros de algodón se encontrase sujeto a competencia monopolística. En casos de competencia monopolística el mercado internacional de un bien no sólo

${ }^{6}$ Sudrià (1987).

7 Rosés (2000), Carreras (1983) y Bardini (1997).

* Tortella (1994).

4 La teoría de los ciclos de aprendizaje de las industrias algodoneras fue avanzada por Sandberg (1968).

10 Indudablemente, si la industria algodonera había superado la fase de «industria naciente» no se puede justificar su protección por esta razón. 
se rige por las leyes de la ventaja comparativa, ya que a mayor mercado interior mayor capacidad exportadora de este tipo de bienes ${ }^{11}$. En este sentido es bien conocido el hecho de que el mercado español de productos textiles fue notablemente más pequeño que otros mercados europeos durante el siglo XIX ${ }^{12}$. No obstante, para que el tamaño del mercado doméstico impida la exportación de un producto concreto es necesario que las economías de escala y aglomeración sean menores en el mercado más pequeño que en el mayor. Por lo tanto, deberíamos preguntarnos por el tamaño relativo de las fábricas algodoneras españolas y de las aglomeraciones españolas de esta industria. Las economías de escala en la producción de artículos algodoneros se agotan con factorías relativamente pequeñas. $\mathrm{Al}$ respecto, Sokoloff demostró que en las empresas algodoneras integradas verticalmente de los Estados Unidos las economías de escala se agotaban hacia los 150 trabajadores ${ }^{13}$. En España, la empresa algodonera integrada verticalmente tenía un tamaño medio de 183 trabajadores en 1850; por tanto, se encontraba más allá de la frontera de las economías de escala de la producción algodonera ${ }^{14}$. Es indudable que la mayor aglomeración de la industria española (Cataluña) era más pequeña que la aglomeración de la industria algodonera en Gran Bretaña (Lancashire), pero, en cambio, era mayor que cualquier aglomeración del sector en Italia. Por ejemplo, en 1876, la región italiana con más husos algodoneros (Piamonte) tenía unos 300.000 , mientras que en Cataluña había por esos años casi el triple de husos ${ }^{15}$. En resumen, el tamaño del mercado interior desempeñó, como mucho, un papel muy secundario en la falta de competitividad de las empresas algodoneras españolas.

La explicación que defiendo en este artículo es que la industria española no exportaba géneros de algodón porque no producía aquellos en los que sí tenía ventaja comparativa internacional, tal como hacía Italia. No todos los tejidos de algodón son iguales y tampoco se producen con la misma función de producción, ya que para producir cada tipo de tejido se utiliza una combinación distinta de factores de producción ${ }^{16}$. Particularmente, para elaborar los tejidos más bastos y baratos se emplea menos trabajo especializado que para producir los tejidos más finos y caros. Con altos

\footnotetext{
11 Krugman (1995).

12 Prados de la Escosura (1983).

${ }^{13}$ Sokoloff (1984).

${ }_{14}$ Rosés (1997).

is A'Hearn (1998) y Nadal (1991).

i6 Harley (1992) y Rosés (2000).
} 
aranceles, tal como sucedía en España ${ }^{17}$, los tejidos se producían de acuerdo a la ventaja comparativa interna porque los precios relativos internacionales no afectaban a los productores nacionales. Es decir, se produciría de acuerdo a los precios relativos de los factores de producción entre las diversas regiones españolas. Así, si Cataluña era relativamente abundante en trabajadores especializados produciría tejidos de algodón que utilicen intensivamente ese factor de producción. Sin embargo, en términos internacionales Cataluña no era abundante en trabajadores especializados, sino escasa. En otras palabras, sus productos no eran competitivos internacionalmente porque Cataluña no producía de acuerdo a su dotación internacional de recursos. De hecho, las clases de tejidos que producía Cataluña, al contrario de lo que sucedía en Italia, se parecían a las clases de tejidos producidas por los británicos. En concreto, la fábricas algodoneras catalanas producían más tejidos de las calidades medias y finas que sus homólogas italianas ${ }^{18}$.

Existía también otra razón para que los productores españoles tratasen de fabricar tejidos de algodón de calidad media y fina en vez de los tejidos más bastos: a medida que la calidad del tejido mejora no sólo aumenta su precio, sino la posibilidad de fabricar un producto diferenciado. En otras palabras, los tejidos de algodón más bastos son relativamente homogéneos y el consumidor puede fácilmente identificar el producto $\mathrm{y}$, por tanto, calcular su valor en el mercado. En cambio, las calidades finas sirven para fabricar productos diferenciados que son difícilmente comparables por el consumidor y que, por tanto, incluyen cierto beneficio monopolístico para el productor. Por esta misma razón, las empresas algodoneras españolas preferían fabricar muchos productos en vez de fabricar un solo tejido y obtener, con ello, economías de escala ${ }^{19}$.

El resto del artículo se ocupa de analizar y probar las hipótesis que hemos expuesto hasta ahora. En la siguiente sección demostraremos que, pese a sus notables progresos durante el período analizado, la industria algodonera española producía a precios más elevados que su homóloga británica. La sección segunda prueba que parte de esa desventaja de precios puede ser atribuida al mayor coste de las materias primas en España; es

${ }_{17}$ La protección arancelaria era mucho menor en la industria algodonera italiana que en la española. Rosés (2000).

${ }^{18}$ Rosés (2000).

${ }_{19}$ En otras palabras, lo que impulsaba a los productores catalanes a fabricar muchos productos diferentes no era el tamaño del mercado y las fluctuaciones de la demanda, tal y como ha sido sugerido por Nadal (1991), sino la posibilidad de obtener esas rentas monopolísticas. 
decir, analiza la cuantía de la «desventaja natural» de los productores españoles de géneros de algodón. La sección tercera compara la productividad de la industria local con la británica y demuestra que la principal razón de la falta de competitividad internacional de la industria algodonera espanola era su ineficiencia.

\section{EL PRECIO DE LOS TEJIDOS ESPAÑOLES EN PERSPECTIVA COMPARADA}

En el caso del hilado de algodón las comparaciones internacionales de precios son simples, ya que éste era un producto relativamente estandarizado. El hilo de algodón se clasificaba con una numeración correlativa que iba desde el más grueso al más fino ${ }^{20}$. El Cuadro 1 compara los precios del hilo de algodón trama «número 30 español» y los correspondientes precios ingleses desde 1830 a 1861.

El Cuadro 1 nos muestra varias cuestiones importantes. En primer lugar, las grandes diferencias entre el precio inglés y el precio éspañol. Sin embargo, este diferencial de precios decreció significativamente a lo largo de los años. En concreto, en 1830 los precios españoles eran un 56 por 100 más altos que los ingleses, mientras que a finales de la década de 1850 esa diferencia se había reducido a la mitad. Debe también señalarse que esta reducción no puede asociarse con los ciclos experimentados por la industria británica o española, movimientos en los tipos de cambios de la libra o la peseta, o la caída del coste de las materias primas en España ${ }^{21}$. Por lo tanto, gran parte de la reducción fue debida a un efecto catching-up, es decir, a un acercamiento de la eficiencia de la industria española a la británica. Otra cuestión importante corresponde a la variabilidad anual de los precios. Mientras que los precios ingleses muestran notables oscilaciones, los españoles permanecen constantes durante periodos relativamente largos ${ }^{22}$. En concreto, sólo en 16 de 29 ocasiones el precio del hilo español se modificó de un año al siguiente. Es indudable

${ }^{20}$ Durante el siglo XIX se utilizaron, al menos, cuatro sistemas diferentes de numeración (inglés, francés, belga y español) y dos tipos de hilo (trama y urdimbre), pero la comparación y conversión entre ellos no supone mayores problemas, ya que Ferrer Vidal (1875) y Ronquillo (1851-1857) ofrecen las equivalencias entre las diferentes medidas.

${ }^{21}$ Véase sobre este último aspecto la próxima sección.

22 En Gran Bretaña había modificaciones de los precios semana a semana siguiendo los cambios en el coste de las materias primas y al movimiento de precios en las bolsas de géneros de algodón. 


\section{CUADRO 1}

Precio del bilo trama del número 30 español en Inglaterra y España (cts. por kg.)

\begin{tabular}{|c|c|c|c|c|c|}
\hline & Inglés & Español & & Inglés & Español \\
\hline $1830 \ldots$. & 284,62 & 656,25 & $1845 \ldots \ldots \ldots \ldots$ & 205,11 & 406,25 \\
\hline $1831 \ldots$ & 240,52 & 625,00 & $1846 \ldots \ldots \ldots \ldots$ & 246,79 & 375,00 \\
\hline $1832 \ldots$. & 270,93 & 593,75 & $1847 \ldots \ldots \ldots \ldots$ & 190,63 & 343,75 \\
\hline $1833 \ldots \ldots \ldots \ldots \ldots$ & 291,56 & 593,75 & $1848 \ldots \ldots \ldots \ldots$ & 181,40 & 329,38 \\
\hline $1834 \ldots \ldots \ldots \ldots \ldots$ & 324,49 & 593,75 & $1849 \ldots \ldots \ldots \ldots$ & 183,08 & 312,50 \\
\hline $1835 \ldots \ldots \ldots \ldots$ & 379,35 & 562,50 & $1850 \ldots \ldots \ldots \ldots$ & 245,18 & 375,00 \\
\hline $1836 \ldots \ldots \ldots \ldots \ldots$ & 366,16 & 562,50 & $1851 \ldots \ldots \ldots \ldots$ & 213,90 & 375,00 \\
\hline $1837 \ldots \ldots \ldots \ldots \ldots$ & 293,28 & 531,25 & $1852 \ldots \ldots \ldots \ldots$ & 214,63 & 343,75 \\
\hline $1838 \ldots \ldots \ldots \ldots \ldots$ & 282,30 & 531,25 & $1853 \ldots \ldots \ldots \ldots$ & 211,40 & 329,38 \\
\hline $1839 \ldots \ldots \ldots \ldots$ & 264,34 & 531,25 & 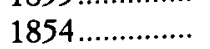 & 192,72 & 375,00 \\
\hline $1840 \ldots \ldots \ldots \ldots$ & 235,91 & 531,25 & $1855 \ldots \ldots \ldots \ldots$ & 196,84 & 343,75 \\
\hline $1841 \ldots \ldots \ldots \ldots \ldots$ & 222,95 & 500,00 & $1856 \ldots \ldots \ldots \ldots$ & 222,91 & 343,75 \\
\hline $1842 \ldots \ldots \ldots \ldots \ldots$ & 211,97 & 437,50 & $1857 \ldots \ldots \ldots \ldots . . . . . . . . .$. & 233,63 & 343,75 \\
\hline $1843 \ldots \ldots \ldots \ldots \ldots$ & 208,29 & 406,25 & $1858 \ldots \ldots \ldots \ldots$ & 256,62 & 343,75 \\
\hline $1844 \ldots \ldots \ldots \ldots \ldots$ & 206,01 & 406,25 & $1859 \ldots \ldots \ldots \ldots$ & 264,52 & 343,75 \\
\hline
\end{tabular}

FUENTE Y NOTAS: Los precios ingleses (números ingleses 30 y 40) son una media aritmética de los precios facilitados por Mann (1968), Economist (1845) (1850) (1855) (1860) y Huberman (1996). La conversión al número 30 español se ha obtenido de acuerdo a la siguiente fórmula: Precio número 30 español $=$ Precio 30 inglés $+0,126 \times$ (precio 40 inglés - precio 30 inglés). Los precios resultantes en chelines se han convertido a pesetas con el tipo de cambio que aparece en Prados de la Escosura (1984). La fuente de los precios españoles es el Diario de Barcelona (1866).

que esto indica que existía poca competencia entre las empresas españolas y es un síntoma de la existencia de acuerdos de precios en el sector ${ }^{23}$.

Los precios de los tejidos de algodón resultan más difíciles de comparar porque éste no es un producto homogéneo. De hecho, los precios de las

${ }^{23}$ Muchas veces se argumenta erróneamente que el número elevado de empresas en el sector evitaba la colusión y permitía la existencia de un mercado competitivo. Rosés (1997). Sin embargo, un numero elevado de empresas no conduce necesariamente a la competencia si cada empresa produce un producto diferenciado, es decir, si produce bajo las condiciones de competencia monopolística; véase al respecto Sutton (1998). En otras palabras, lo que no importa es el número total de empresas en el sector, sino cuántas empresas producen en cada segmento del mercado. Como la industria algodonera tiene numerosos nichos de mercado, las posibilidades de colusión u oligopolio son muchas. De hecho, esto explicaría la tendencia de muchas empresas a evitar los mercados intermedios y a integrar verticalmente la producción de todas las fases. 


\section{CUADRO 2}

Precios de diferentes clases de tejidos crudos, 1860 (ptas.)

\begin{tabular}{|c|c|c|c|}
\hline & $\begin{array}{c}\text { Densidad } \\
\mathrm{Gr} / \mathrm{m}^{2}\end{array}$ & $\begin{array}{l}\text { Precio } \\
\text { Ptas } / \mathrm{kg}\end{array}$ & $\begin{array}{l}\text { Precio } \\
\text { Ptas } / m^{2}\end{array}$ \\
\hline \multicolumn{4}{|l|}{ Inglés } \\
\hline 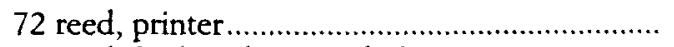 & 128 & 3,57 & 0,46 \\
\hline 48 reed, Red End Long Cloth... & 125 & 2,64 & 0,33 \\
\hline 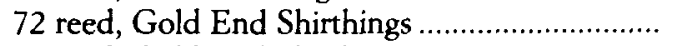 & 121 & 3,47 & 0,42 \\
\hline 66 reed, Gold End Shirthings ............................. & 114 & 3,32 & 0,38 \\
\hline 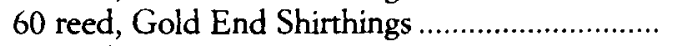 & 110 & 3,13 & 0,35 \\
\hline 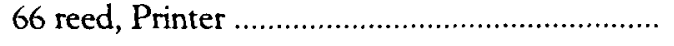 & 107 & 3,97 & 0,42 \\
\hline \multicolumn{4}{|l|}{ Español } \\
\hline 80 hilos, 5/4 2.. & 124 & 5,90 & 0,73 \\
\hline 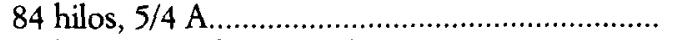 & 122 & 6,26 & 0,77 \\
\hline 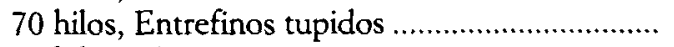 & 121 & 5,17 & 0,62 \\
\hline 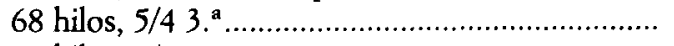 & 120 & 5,35 & 0,64 \\
\hline 66 hilos, 7/8 1. a & 113 & 4,93 & 0,56 \\
\hline 60 hilos, $7 / 8$ 2. & 102 & 4,50 & 0,46 \\
\hline
\end{tabular}

FUENTES: La fuente de los precios ingleses es Economist (1860) y todos los precios son de enero de 1860 mientras que la fuente de los precios españoles es Comisión Especial Arancelaria (1867). Todos los precios españoles proceden de la respuesta de José Ferrer y Cía., excepto en el caso del precio de «Entrefinos tupidos», que procede de la respuesta a la comisión de la España Industrial, S. A. Los productos españoles fueron escogidos de acuerdo a su semejanza con los productos ingleses de los que he conseguido precios. Los precios británicos fueron convertidos en pesetas con el típo de cambio de Prados de la Escosura (1994). Las cifras se encuentran sujetas a pequeños errores por redondeo.

piezas pueden variar por cambios en la calidad del hilado, el tamaño de la pieza, el número de hilos por pulgada (la densidad del tejido), y el tipo de acabado. El Cuadro 2 ilustra las dificultades para comparar los precios de los tejidos de algodón.

Es realmente difícil encontrar en la lista de tejidos que aparece en la tabla dos completamente iguales. Sólo el «72 reed, Gold End Shirthings» y el «70 hilos, entrefinos tupidos» eran prácticamente iguales. Es también posible comparar el precio del tejido «66 reed, Printer» con un tejido español hipotético que es una media de los precios del «66 hilos, 7/8 1. ${ }^{a} »$ y el $\ll 66$ hilos, $7 / 8$ 2. ${ }^{a} »$ (dos tejidos muy similares). En el primer caso, el tejido inglés costaba un 60 por 100 del precio del español, y en el segundo, un 80 por 100 . Es importante resaltar que esas cifras también 


\section{GRÁFICO 1}

Precios del tejido crudo para pintar, 1830-1860 (Cts. por $\left.\mathrm{m}^{2}\right)$

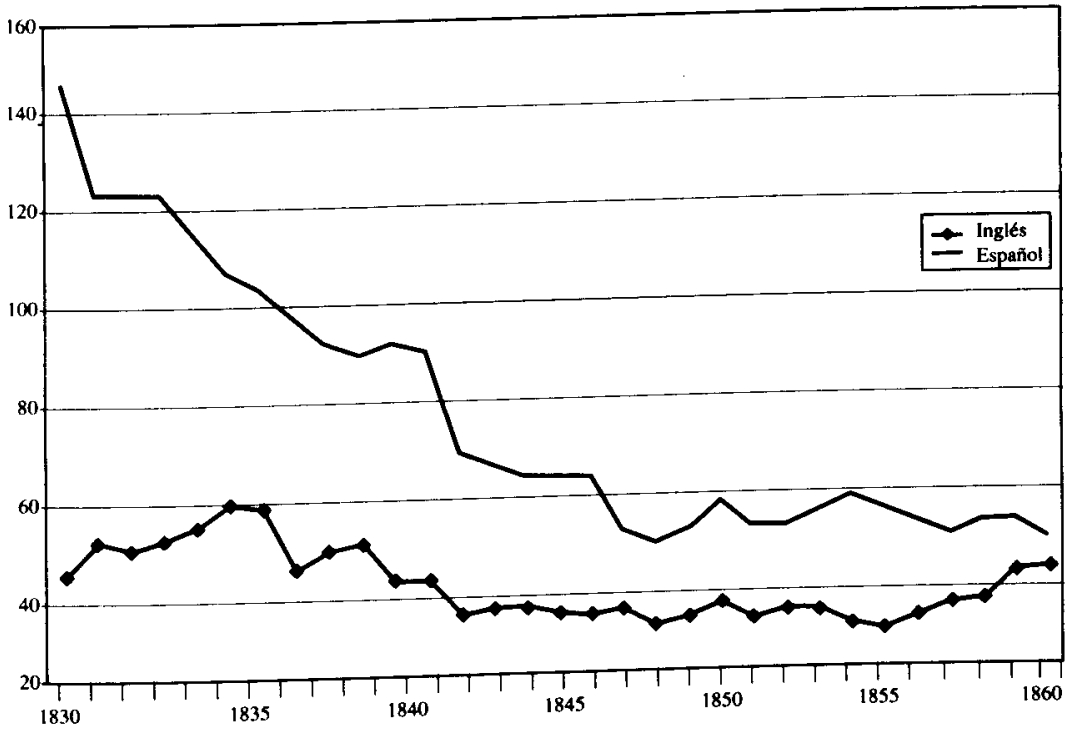

FUENTES Y NOTAS: Los precios ingleses corresponden al tejido «66 reed printer» hasta 1845, mientras que desde 1830 hasta 1845 las series son las del « 72 reed printerm, la única disponible (ambas series han sido enlazadas para evitar desviaciones). La fuente de los datos desde 1845 es Economist (1845) (1850) (1855) (1960) y en las series anteriores Blaug (1961), p. 376. Las series españolas son una media aritmética de los precios de los tejidos «66 hilos 7/8 1. ${ }^{\mathrm{a}}$ » « 60 hilos 7/8 2. ${ }^{\mathrm{a}} »$ tal como aparecen en Comisión Especial arancelaria (1867). Esos precios corresponden exactamente con el precio máximo y mínimo, respectivamente, de las series de precios con el título «Tejido crudo para pintar» que aparecen en el Diario de Barcelona (1866). Como en ocasiones anteriores, los precios británicos fueron convertidos en pesetas con el tipo de cambio de Prados de la Escosura (1984).

muestran que la diferencia de precios entre británicos y españoles crecían a medida que los tejidos eran más finos.

El Gráfico 1 muestra la diferencia de precios entre el tejido crudo inglés y el español desde 1830 a 1860. Dos hechos llaman nuestra atención. En primer lugar, la diferencia de precio entre el tejido español y el británico era muy grande aunque se redujo de manera considerable durante el período. En concreto, en la década de 1830 los precios españoles doblaban a los británicos, mientras que al final del período considerado la diferencia se había reducido a un 25 por 100 . En segundo lugar, tal como sucedía 
con el precio de los hilados, los precios españoles fluctuaban anualmente mucho menos que los ingleses.

En resumen, a lo largo de esta sección hemos establecido que el precio de los hilados y tejidos de algodón era mucho mayor en España que en Gran Bretaña. Sin embargo, esta diferencia de precios se fue reduciendo paulatinamente pero sin llegar a converger completamente, ya que siempre los productos españoles fueron al menos una cuarta parte más caros que los británicos. En las siguientes dos secciones vamos a analizar el porqué de este sobreprecio de los algodones nacionales.

\section{EL COSTE DE LAS MATERIAS PRIMAS}

La materia prima más importante en la fabricación de géneros de algodón es, obviamente, el algodón en rama. En el período 1830-1860, los Estados esclavistas del sur de los Estados Unidos fueron sus principales productores. No obstante el dominio norteamericano, el algodón también era cultivado en Egipto, Brasil, algunas islas caribeñas y la India. El mayor mercado mundial al por mayor de algodón en rama se situaba en Liverpool cerca del Lancashire, la mayor concentración mundial de fábricas de géneros de algodón ${ }^{24}$. En España, la mayoría de las importaciones de algodón tenían lugar a través del puerto de Barcelona. Las fábricas catalanas compraban el algodón en Liverpool, en Nueva Orleans o a los mayoristas del puerto catalán, que a su vez lo adquirian directamente de las zonas productoras o en Liverpool ${ }^{25}$.

El Cuadro 3 muestra el coste de algodón norteamericano de la misma calidad en los puertos de Liverpool y Barcelona. Los precios son al por mayor e incluyen el coste de los fletes, del acarreo de los barcos al puerto, los aranceles y tasas, y todos los demás costes menores. En España existían aranceles sobre las importaciones de algodón que trataban de incentivar las importaciones directas del algodón en rama en buques españoles e incluían un derecho diferencial de bandera ${ }^{26}$.

Debe resaltarse que los precios tienden a fluctuar más severamente en los mercados pequeños que en los mercados grandes; por eso cabría (1968).

${ }^{24}$ Véase sobre el mercado internacional del algodón en rama, Mann (1968) y Ellison

${ }^{25}$ Comisión Especial Arancelaria (1867).

${ }^{26}$ Concretamente, a finales de los años 1850 , los aranceles eran de $7 \mathrm{Rv}$. por Qm. si el buque era español y procedía de las zonas productores, y de $34 \mathrm{Rv}$. por Qm. si el buque procedía de puerto de depósito (Liverpool, por ejemplo). En buques extranjeros 


\section{CUADRO 3}

Precios del algodón US Middling en Liverpool y Barcelona, 1830-1860 (cts. por kg.)

\begin{tabular}{|c|c|c|c|c|c|}
\hline & Liverpool & Barcelona & & Liverpool & Barcelona \\
\hline $1830 \ldots \ldots \ldots \ldots \ldots$ & 157 & 188 & $1845 \ldots \ldots \ldots \ldots$ & 96 & 121 \\
\hline $1831 \ldots \ldots \ldots \ldots \ldots$ & 128 & 192 & $1846 \ldots \ldots \ldots \ldots . . . .$. & 112 & 136 \\
\hline $1832 \ldots \ldots \ldots \ldots \ldots$ & 158 & 182 & $1847 \ldots \ldots \ldots \ldots$ & 140 & 168 \\
\hline 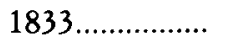 & 196 & 199 & $1848 \ldots \ldots \ldots \ldots$ & 97 & 125 \\
\hline 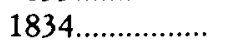 & 198 & 239 & $1849 \ldots \ldots \ldots \ldots$ & 93 & 146 \\
\hline $1835 \ldots \ldots \ldots \ldots \ldots$ & 226 & 273 & $1850 \ldots \ldots \ldots \ldots$ & 158 & 188 \\
\hline $1836 \ldots \ldots \ldots \ldots \ldots$ & 214 & 257 & $1851 \ldots \ldots \ldots \ldots$ & 122 & 188 \\
\hline $1837 \ldots \ldots \ldots \ldots$ & 141 & 227 & $1852 \ldots \ldots \ldots \ldots$ & 112 & 156 \\
\hline $1838 \ldots \ldots \ldots \ldots$ & 150 & 184 & $1853 \ldots \ldots \ldots \ldots$ & 122 & 163 \\
\hline $1839 \ldots \ldots \ldots \ldots$ & 175 & 212 & $1854 \ldots \ldots \ldots \ldots$ & 117 & 158 \\
\hline $1840 \ldots \ldots \ldots \ldots$ & 128 & 175 & $1855 \ldots \ldots \ldots \ldots$ & 124 & 165 \\
\hline $1841 \ldots \ldots \ldots \ldots \ldots$ & 138 & 155 & $1856 \ldots \ldots \ldots \ldots . . . . .$. & 138 & 164 \\
\hline $1842 \ldots \ldots \ldots \ldots$ & 116 & 134 & $1857 \ldots \ldots \ldots \ldots$ & 173 & 188 \\
\hline $1843 \ldots \ldots \ldots \ldots$ & 105 & 133 & $1858 \ldots \ldots \ldots \ldots$ & 157 & 200 \\
\hline $1844 \ldots \ldots \ldots \ldots \ldots$ & 113 & 166 & $1859 \ldots \ldots \ldots \ldots$ & 149 & 189 \\
\hline
\end{tabular}

FuENTES: Las fuentes de precios de Liverpool es Harley (1992) mientras que la fuente de los precios españoles es Diario de Barcelona (1866). Los precios ingleses en libras se han convertido a pesetas con el tipo de cambio que aparece en Prados de la Escosura (1984).

esperar que las fluctuaciones de los precios en Barcelona fuesen mayores que en Liverpool. Los precios de Barcelona durante el período no convergieron con los de Liverpool, la diferencia se mantuvo alrededor de un 28 por 100 . Además, una contabilidad detallada de los costes de importación que afectaban al precio del algodón en rama en Barcelona muestra que los dos principales componentes del sobreprecio del algodón en Barcelona eran los fletes (que ascendían a más del 62 por 100 del sobreprecio) y el arancel español sobre las importaciones de algodón en rama (que representaba otro 25 por 100) ${ }^{27}$.

El segundo coste más importante para las fábricas catalanas, después del algodón en rama, era el carbón. En esta época, las fábricas algodoneras

los aranceles crecian hasta los $44 \mathrm{Rv}$. por Qm. y los $52 \mathrm{Rv}$. por Qm. respectivamente. Comisión Especial Arancelaria (1867).

${ }_{27}$ Comisión Especial Arancelaria (1867). 
eran movidas por caballos, maquinas de vapor y ruedas hidráulicas. En cambio, la mayoría de las fábricas británicas empleaban la fuerza del vapor ${ }^{28}$. En Cataluña el coste del carbón era mayor que en Gran Bretaña, ya que, en esta época, importaba todo el carbón que consumía. Por eso, el alto coste del combustible retrasó el triunfo en Cataluña del vapor sobre otras fuentes de energía ${ }^{29}$. Por ejemplo, el Censo de Sayró muestra que en 1840 solamente una pequeña parte de toda la maquinaria de hilar era movida por máquinas de vapor o ruedas hidráulicas, mientras que la gran mayoría de los telares eran aún manuales ${ }^{30}$. No obstante, esta situación se alteró notablemente en poco tiempo porque al final de los años 1850 al menos tres cuartas partes de los husos de hilar y la mitad de los telares de la industria algodonera ya eran movidos por máquinas de vapor ${ }^{31}$. Es probable que el rápido triunfo de la máquina de vapor fuese debido a la adopción de las nuevas máquinas de alta presión que ahorraban gran cantidad de carbón y, en consecuencia, eran mucho más rentables dadas las condiciones económicas de Cataluña. La mayor parte del carbón utilizado por las fábricas catalanas llegaba a sus costas por medio de veleros que lo traían directamente desde Gran Bretaña, principalmente de los puertos de Cardiff y Newcastle. Los altos costes del flete (el carbón es una mercancía barata pero pesada) y los aranceles incrementaban el precio del carbón en un 76 por 100 en el trayecto desde las costas británicas hasta Barcelona ${ }^{32}$.

El coste del algodón en rama y del carbón pueden ayudarnos a aproximarnos a la ausencia de competitividad de la industria algodonera catalana en los mercados internacionales. En el Cuadro 4, se presenta una estimación de la parte de la diferencia de precios entre los productores españoles y los ingleses que puede ser explicada por el alto coste de las materias primas en Cataluña. Para este análisis, se supone que las factorías catalanas no modifican su función de producción con los cambios en los precios relativos de los inputs materiales. Podría argumentarse que este supuesto puede ser muy irreal, ya que el mayor coste del combustible y del algodón en rama hacía que los españoles ahorrasen más en estos bienes que sus competidores británicos. Es probable, sin embargo, que las diferencias en el precio de las materias primas afectasen más a la clase de tejidos que se fabricaban que a la cantidad de inputs empleados para fabricar cada

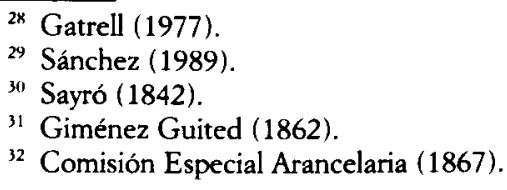




\section{CUADRO 4}

Las fuentes del sobreprecio de los tejidos de algodón españoles, $1845-1858$

\begin{tabular}{|c|c|c|c|}
\hline & $\begin{array}{c}\text { Diferencia } \\
\text { de precios } \\
\left(\mathrm{cts} / \mathrm{m}^{2}\right)\end{array}$ & $\begin{array}{c}\text { Por materias } \\
\text { primas } \\
\left(\mathrm{cts} / \mathrm{m}^{2}\right)\end{array}$ & $\begin{array}{l}\text { Porcentaje } \\
\text { explicado }\end{array}$ \\
\hline 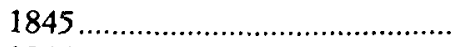 & 27,78 & 8,47 & 30,51 \\
\hline 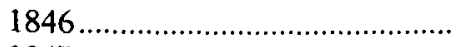 & 28,37 & 8,36 & 29,45 \\
\hline (........................... & 15,73 & 8,81 & 56,05 \\
\hline $1848 \ldots \ldots \ldots \ldots \ldots \ldots$ & 16,75 & 8,77 & 52,38 \\
\hline $1849 \ldots \ldots$ & 17,70 & 12,00 & 67,78 \\
\hline 1850 & 21,05 & 9,00 & 42,76 \\
\hline 1851 & 18,57 & 13,65 & 73,52 \\
\hline 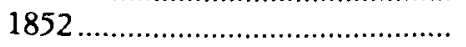 & 16,97 & 10,82 & 63,77 \\
\hline 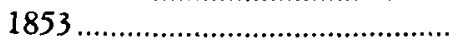 & 20,20 & 10,46 & 51,75 \\
\hline 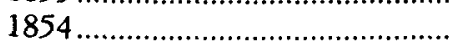 & 26,06 & 10,44 & 40,08 \\
\hline 1855 & 24,69 & 10,50 & 42,51 \\
\hline 1856 & 19,18 & 8,50 & 44,32 \\
\hline 1857 & 13,95 & 7,18 & 51,47 \\
\hline 1858 & 15,95 & 10,75 & 67,39 \\
\hline Media............... & 20,21 & 9,84 & 50,98 \\
\hline
\end{tabular}

FUENTES Y NOTAS: Este diferencial de precios ha sido calculado con los datos del Cuadro 3 y del Gráfico 1. En las materias primas ha sido incluida la contribución industrial porque un impuesto similar no existía en Gran Bretaña. De acuerdo con los datos que aparecen en Comisión Especial Arancelaria (1867), la cantidad de carbón empleada en producir un metro cuadrado de este tipo de tejido era de $0,55 \mathrm{~kg}$, la cantidad de algodón en rama era de $0,126376 \mathrm{~kg}$., y la contribución industrial ascendía a $2,8791 \mathrm{cts}$.

clase de tejidos. Esto es, cada clase de tejidos era fabricada con una proporción constante de materias primas. Por ejemplo, las fábricas algodoneras españolas y británicas empleaban la misma cantidad y tipo de algodón para producir cada calidad de tejido. Además, las fábricas catalanas copiaron las velocidades a las que trabajaban las fábricas británicas; es decir, consumían la misma cantidad de carbón para producir cada tipo de tejido. Finalmente, quiero resaltar que los cálculos suponen que las fábricas británicas y catalanas se encontraban situadas en los mismos puertos donde se producían las importaciones de materias primas y donde su precio era menor. El hecho de que una parte importante de la industria catalana se encontrara en el puerto de Barcelona mientras que la británica se encontrase alejada de Liverpool puede exagerar la parte de la diferencia de los precios que es explicada por el mayor coste de los materiales en Cataluña. 
Las cifras del Cuadro 4 son esclarecedoras, ya que, aproximadamente, la mitad del sobreprecio de los tejidos españoles puede ser explicado por el mayor coste de las materias primas. En otras palabras, la falta de competitividad de las empresas algodoneras españolas en los mercados internacionales no puede atribuirse completamente a desventajas naturales $y$, por tanto, es lógico que una buena parte deba imputarse a su menor eficiencia respecto a la británica. Precisamente, en la próxima sección vamos a discutir en detalle este extremo.

\section{LA EFICIENCIA RELATIVA DE LA INDUSTRIA ALGODONERA CATALANA EN 1850}

Para calcular la eficiencia relativa de la industria algodonera catalana en 1850 vamos a utilizar el modelo sugerido por Allen, que permite descomponer el coste relativo del valor añadido unitario de los tejidos de un país respecto a otro en productividad total de los factores (PTF) o eficiencia técnica relativa, eficiencia relativa del mercado, y diferencias en el precio de los componentes del valor añadido (trabajo y capital) ${ }^{33}$. La principal ventaja de este modelo sobre otros utilizados comúnmente en esta clase de estudios comparativos es que se adapta a situaciones en que en alguno de los países analizados no hay competencia perfecta. Concretamente, en este caso, todos nuestros cálculos parten del supuesto de que Gran Bretaña produce de manera competitiva mientras que en Cataluña existe competencia imperfecta. La ecuación básica del modelo es:

Coste de una unidad de valor añadido = Eficiencia técnica (PTF)

$\times$ Eficiencia del mercado $\times$ Precio relativo del capital y trabajo,

es decir, de forma algebraica, en el caso de dos países:

$$
\frac{w^{1} \cdot x^{1}}{w^{0} \cdot x^{0}}=\frac{1}{u} \cdot \frac{w^{1} \cdot x^{2}}{w^{1} \cdot x^{3}} \cdot \frac{c\left(w^{1}\right)}{c\left(w^{0}\right)}
$$

33 Clark (1987) realizó un estudio comparado de la eficiencia de las industrias algodoneras de varios países, entre ellos España, para principios del siglo xx. En el modelo de Allen (1991) se parte del supuesto que capital y trabajo se combinan para producir el valor añadido, y que los dos países utilizan las mismas cantidades de materiales para cada tipo de tejido, tal como en realidad sucedía. 
donde la PTF $(1 / u)$ se calcula, suponiendo tecnología Cobb-Douglas ${ }^{34}$, como:

$$
u=\frac{Q^{1} / Q^{0}}{\prod_{i=1}^{n}\left(\frac{Z_{i}^{1}}{Z_{i}^{0}}\right)^{S_{i}^{0}}} ; S_{i}^{0}=W_{i}^{0} Z_{i}^{0} / \sum_{i=1}^{n} W_{i}^{0} Z_{i}^{0}
$$

donde el precio relativo del capital y del trabajo $C\left(W^{1}\right) / C\left(W^{0}\right)$ se determina con la siguiente ecuación:

$$
\frac{C\left(W^{1}\right)}{C\left(W^{0}\right)}=\prod_{i=1}^{n}\left(\frac{W_{i}^{1}}{W_{i}^{0}}\right)^{S_{i}^{0}} ; S_{i}^{0}=W_{i}^{0} Z_{i}^{0} / \sum_{i=1}^{n} W_{i}^{0} Z_{i}^{0}
$$

donde $Q$ se refiere al output, $W_{i}$ a las cantidades de inputs, $Z_{i}$ a su precio, y 0 es Gran Bretaña mientras que 1 es Cataluña. El coste de una unidad de valor añadido se calcula directamente de las fuentes dividiendo el coste del valor añadido, es decir, la remuneración del capital y del trabajo, entre la cantidad de producto. Asimismo, la eficiencia relativa de los mercados se puede hallar resolviendo la ecuación (1a) una vez que conocemos las otras tres variables.

El primer paso para calcular la eficiencia relativa de las industrias algodoneras catalana y británica consiste en la construcción de medidas homogéneas del producto de sus fábricas. Sin embargo, debemos eliminar de estas cifras las cantidades de hilo que las industrias catalana y británica exportaban a otros países o sectores. Los británicos exportaban una parte de su producción de hilo a otros países y otra parte importante a los tejedores manuales. La industria catalana exportaba hilo a la industria de tejidos de mezcla y también una parte a los tejedores manuales de algodón. En el caso británico, siguiendo a Von Tunzelmann, se supone que todo el

${ }^{34}$ Allen (1991) demuestra que la forma de la función de producción es esencial para los cálculos de la productividad relativa. Sin embargo, en el caso de la industria algodonera, esto no parece un problema grave, ya que diversos estudios, por ejemplo, Sokoloff (1984), señalan que en esta industria se produce de acuerdo a la función Cobb-Douglas. 
hilo de más calidad era utilizado por los tejedores manuales y que las exportaciones internacionales se distribuían uniformemente entre las diferentes calidades ${ }^{35}$. En el caso catalán, asumimos que el hilo producido por las 'selfactinas' y las 'tbrostles' era usado para el tejido mecánico y el hilado de las 'mule-jennies' se vendía a los tejedores manuales. Este supuesto es plenamente corroborado por los censos industriales donde las empresas que integran verticalmente hilado y tejido mecánicos emplean solamente 'throstles' y 'selfactinas' ${ }^{36}$. También para Cataluña se ha supuesto que los telares mecánicos fabricaron un 80 por 100 de la calidad G2 y un 20 por 100 de la calidad GF. En segundo lugar, debemos desglosar el producto en categorías homogéneas. En concreto, de acuerdo a la clasificación propuesta por Huberman, vamos a desagregar los tejidos de algodón en cinco calidades (de la más gruesa a la más fina, G1, G2, GF, F1 y F2) ${ }^{37}$. Finalmente, el producto es convertido a metros cuadrados equivalentes de la calidad más basta (G1), usando los precios relativos de los diversos tejidos en Gran Bretaña. Con este método obtenemos una medida del producto que tiene en cuenta las diferencias de calidad y la falta de competencia en el seno de la industria catalana. El Cuadro 5 presenta los resultados de todos estos cálculos.

Las dos primeras secciones del Cuadro 6 presentan los totales de mano de obra y capital en las fábricas algodoneras. Los datos británicos han sido obtenidos de Blaug y Von Tunzelmann mientras que los catalanes proceden del Censo de la Junta de Fábricas y comprenden todos los trabajadores en 'throstles', 'selfactinas' y telares mecánicos ${ }^{38}$. Las cifras de trabajadores han sido convertidas en trabajadores masculinos equivalentes y horas equivalentes. Ante le falta de datos sobre el salario relativo de los niños en Gran Bretaña se han utilizado las cifras catalanas para proceder a la conversión de trabajadores masculinos, femeninos y niños en trabajadores masculinos equivalentes ${ }^{39}$. El número de trabajadores masculinos equivalentes también ha sido ajustado por las diferencias en las horas trabajadas al año. En Gran Bretaña, las jornadas en las fábricas duraban diez horas, por limitación legal, y se trabajaba durante trescientos días

3s Von Tunzelmann (1978).

36 Junta de Fábricas (1850).

${ }^{37}$ Huberman (1996).

3* Blaug (1961), Von Tunzelmann (1978) y Junta de Fábricas (1850).

${ }^{39}$ La conversión en trabajadores masculinos equivalentes se realiza multiplicando el número de trabajadores femeninos e infantiles por su salario relativo, que es, en este caso, su salario medio partido por el salario medio de los hombres. 


\section{CUADRO 5}

La producción de las fábricas algodoneras, 1850

\begin{tabular}{lccccc}
\hline & $G 1$ & $G 2$ & $G F$ & $F 1$ & $\begin{array}{c}\text { G1 } \\
\text { Equivalente }\end{array}$ \\
\hline Precio relativo... & 1 & 1,25 & 1,4748 & 4,973 & - \\
Cataluña.......... & - & 21.929 & 5.482 & - & 35.496 \\
Gran Bretaña ... & 173.536 & 619.398 & 376.820 & 96.072 & 1.981 .284 \\
\hline
\end{tabular}

FUENTES Y NOTAS: Las cifras en miles de metros cuadrados. Para una descripción detallada de las calidades y los métodos de estimación de las mismas véase Huberman (1996) para Gran Bretaña, y Rosés (2000), para Cataluña.

al año mientras que en Cataluña la jornada normal era de 11,5 horas, pero sólo se trabajaba durante doscientos setenta y siete días ${ }^{40}$. Según los contemporáneos, estas largas paradas de las fábricas catalanas eran consecuencia de la imposibilidad de trabajar durante los días más cálidos del verano, de las fluctuaciones del mercado y de las demoras en la recepción de piezas de repuesto ${ }^{41}$.

La comparación directa de la cantidad de capital entre los dos países puede conducir a errores debido a las enormes diferencias en los precios de los bienes de equipo entre Cataluña y Gran Bretaña ${ }^{42}$. En consecuencia, se ha optado por utilizar una medida cuántica del capital (husos mecánicos equivalentes) que comprende los husos mecánicos más las fuentes de energía y los telares mecánicos. Las cifras españolas fueron obtenidas directamente del Censo de la Junta de Fábricas (1850) y corresponden al número de 'selfactinas' y 'throstles'. Las cifras británicas proceden de Blaug, restando a la cantidad el 20 por 100 de husos de doblar y el porcentaje de husos que trabajaban para el sector exportador tal como fue definido con anterioridad ${ }^{43}$. Finalmente, para ajustar por el menor número anual de horas (1968)

40 Los datos de Gran Bretaña proceden de Ellison (1968) y los de Cataluña de Cerdá

${ }^{41}$ Cerdá (1968) y Ferrer Vidal (1875)

42 En concreto, Chadwick estimó que en 1860 el capital fijo invertido en cada nuevo huso en Gran Bretaña era de 24 chelines, y en cada nuevo telar mecánico, de 24 libras. Suponiendo que cada telar mecánico consumía la producción de 45 husos, el coste agregado de un huso con la parte correspondiente de telar mecánico era de 34,6 chelines. Blaug (1961), p. 373. En cambio, en Cataluña, según la Comisión Especial Arancelaria (1867) el coste del capital fijo asociado (incluyendo su parte de telar) a un huso mecánico era de 112,85 pesetas ( 95 chelines).

${ }^{43}$ Blaug (1961). 


\section{CUADRO 6}

La mano de obra, el capital, el valor añadido, y los costes unitarios en las fábricas algodoneras británicas y catalanas, 1850

\begin{tabular}{|c|c|c|c|c|c|c|}
\hline Trabajo & & Hombres & Mujeres & Niños & $\begin{array}{c}\text { Hombres } \\
\text { Equivalentes }\end{array}$ & $\begin{array}{c}\text { Horas } \\
\text { Equivalentes }\end{array}$ \\
\hline Cataluña............. & & 3.350 & 3.282 & 1.883 & 5.457 & 17.382 .987 \\
\hline Gran Bretaña .... & & 106.368 & 147.956 & 12.263 & 184.646 & 553.939 .293 \\
\hline Capital & & & & & $\begin{array}{c}\text { Husos } \\
\text { Equivalentes }\end{array}$ & $\begin{array}{l}\text { Millones } \\
\text { de horas }\end{array}$ \\
\hline Cataluña .............. & & & & & 147.368 & 469 \\
\hline Gran Bretaña .... & & & & & 10.852 .929 & 32.559 \\
\hline Remuneración & $\begin{array}{l}\text { Capital } \\
\text { fijo }\end{array}$ & Existencias & $\begin{array}{c}\text { Renta } \\
\text { del capital }\end{array}$ & Salarios & $\begin{array}{c}\text { Valor } \\
\text { Añadido }\end{array}$ & $\begin{array}{c}\text { Costes } \\
\text { unitarios }\end{array}$ \\
\hline Cataluña.............. & 827 & 558 & 144 & 168 & 313 & 8,825 \\
\hline Gran Bretaña .... & 18.775 & 8.782 & 2.457 & 7.990 & 10.447 & 5,273 \\
\hline
\end{tabular}

FUENTES y NOTAS: Véase el texto y notas al pie. Todos los valores de la remuneración en miles de libras, excepto los costes unitarios que aparecen en libras [las pesetas han sido convertidas al cambio de 24,32 pesetas por libra que aparece en Prados de la Escosura (1984) para el año 1850]. El coste unitario es el resultado de dividir el valor añadido entre el output en metros cuadrados equivalentes de la calidad G1 tal como aparece en el Cuadro 5.

de trabajo en Cataluña los husos equivalentes fueron multiplicados por las horas de trabajo de las fábricas en cada país.

La última fase para poder determinar la eficiencia relativa de las industrias catalana y británica consiste en estimar el coste del capital y del trabajo, es decir, el valor añadido, de la industria algodonera. La renta del capital que hemos calculado es competitiva; es decir, no se ha considerado para su cómputo la remuneración de las rentas procedentes de los aranceles y se ha supuesto en ambos países la misma tasa de depreciación ${ }^{44}$. Los salarios británicos proceden de Wood mientras que los catalanes son una

${ }^{44}$ En concreto, la formula utilizada ha sido $R_{i}=P_{i}(t) \times r+P_{i}(t) \times \gamma$, donde la renta del capital es igual al precio multiplicado por la tasa de beneficio más el precio mul. tiplicado por la tasa de depreciación. El valor del capital fijo se ha obtenido multiplicando el número de husos por su precio. También se han estimado el valor de las existencias con los datos de Blaug (1961) y Ronquillo (1851-1857). Las tasas de depreciación son del 7,5 por 100 en maquinaria, del 2,5 por 100 en edificios, y del 0 por 100 en existencias. Las tasa de beneficio (tasa de interés más 1 por 100) se ha calculado igual al 5 por 100 en Gran Bretaña y al 7 por 100 en Cataluña. 
media aritmética de las cifras de 1840 y $1860^{45}$. Los resultados de todos estos cálculos aparecen en la tercera sección del Cuadro 6.

El Cuadro 7 presenta la eficiencia comparada de las factorías algodoneras catalanas respecto a las británicas siguiendo el modelo expuesto al principio de esta sección. Es decir, las diferencias en los costes unitarios relativos se descomponen en ineficiencia técnica, ineficiencias del mercado y diferencias en el precio de los inputs. Valores superiores a uno indican que Cataluña es más ineficiente que Gran Bretaña mientras que valores inferiores a uno vendrían a señalar lo contrario.

El Cuadro 7 confirma la hipótesis que habíamos avanzado al final de la sección previa: una parte importante de las diferencias de precio entre las industrias española y británica no se puede explicar por las diferencias de coste de las materias primas a favor de la segunda, sino por la menor eficiencia de la industria española con respecto a la británica. Los costes unitarios son hasta un 67 por 100 mayores en Cataluña que en Gran Bretaña; es decir, para producir cada unidad de output los catalanes emplean hasta un 67 por 100 más de capital y trabajo. Esta enorme diferencia no se puede explicar por el mayor coste del capital en España, ya que las diferencias del precio de los inputs sólo son de cerca del 3 por 100. Asimismo, la ineficiencia de los mercados es mayor en España que en Gran Bretaña alcanzando una cifra cercana al 12 por 100. Sin embargo, éste tampoco es el factor principal cuando pretendemos explicar los mayores costes unitarios españoles, ya que, de hecho, éstos se explican principalmente por la gran ineficiencia técnica de la industria algodonera catalana, casi del 46 por 100. En resumen, a la luz de todo lo expuesto en esta sección y la anterior, la falta de competitividad internacional de la industria algodonera nacional no se puede achacar a las desventajas naturales de España ni a la menor eficacia de los mercados españoles, sino que debe explicarse a partir de razones internas a la misma industria.

¿Qué razones podrían causar la menor productividad total de los factores de las fábricas algodoneras catalanas? Como ya hemos avanzado con anterioridad, esta ineficiencia técnica de la industria española no puede ligarse a su atraso tecnológico, ya que las fábricas catalanas empleaban la misma maquinaría y métodos que sus homólogas británicas. Bien al contrario, la razón principal de la baja PTF radica en que los empresarios catalanes fabricaban productos que se encontraban fuera de su ventaja

${ }^{45}$ Para los salarios británicos, Blaug (1961); para 1840, Madoz (1846), y para 1860, Comisión Especial Arancelaria (1867). 


\section{CUADRO 7}

Costes unitarios relativos, ineficiencias técnicas y del mercado y diferencias del precio de los inputs en las industrias algodoneras de Cataluña y Gran Bretaña (Gran Bretaña $=1$ )

\begin{tabular}{cccc}
\hline $\begin{array}{c}\text { Costes unitarios } \\
\text { relativos }\end{array}$ & $\begin{array}{c}\text { Ineficacia técnica } \\
(P T F)\end{array}$ & $\begin{array}{c}\text { Ineficiencias } \\
\text { del mercado }\end{array}$ & $\begin{array}{c}\text { Diferencias } \\
\text { en el precio } \\
\text { de los inputs }\end{array}$ \\
\hline 1,673 & 1,458 & 1,116 & 1,028 \\
\hline
\end{tabular}

FUENTES Y NOTAS: Los datos proceden del Cuadro 6. Los costes unitarios relativos son el resultado de dividir los costes catalanes entre los británicos. La ineficiencia técnica fue calculada como $1 / u$ siguiendo la ecuación (2). Las ineficiencia del mercado fue estimada como residual según la ecuación (1b). Las diferencias del precio de los inputs fueron evaluadas según la ecuación (3). En todos los cálculos Gran Bretaña es 0 y Cataluña 1. Por tanto, las cifras son valores catalanes respecto a los valores británicos.

comparativa en un segmento del mercado donde no optimizaban su dotación internacional de recursos. En otras palabras, producían bienes que se encontraban por encima de la eficiencia de sus trabajadores. Así, la industria algodonera española fabricaba principalmente tejidos medios y finos (aquellos con una mayor densidad) tal como hacían los británicos ${ }^{46}$. El Gráfico 2 nos muestra cómo afectaba esta elección a las diferencias de precios entre Gran Bretaña y España.

El Gráfico 2 es bastante elocuente: los productos españoles son menos competitivos a medida que crece la calidad, es decir, la densidad del tejido, porque aumenta la participación del trabajo y del capital en el precio final. Por ejemplo, en la calidad más gruesa el capital y el trabajo representan, en España, un 37 por 100 de los costes, mientras que en la calidad más fina que se fabricaba en España esta cifra asciende hasta el 66 por $100^{47}$. En otras palabras, las fábricas españolas se encontraban produciendo por encima de su nivel de eficiencia utilizando una cantidad excesiva de capital y trabajo.

En la fabricación de géneros de algodón la «calidad» de la mano de obra influye grandemente en su productividad. Los tejidos más finos sufren el problema de que los hilos se rompen más, requiriendo por ello una

46 Los británicos producían incluso más fino que los españoles porque en España no se hilaba por encima del número 60 mientras que en Gran Bretaña sí (Rosés, 2000).

${ }^{47}$ Rosés (2000). 


\section{GRÁFICO 2}

Los precios de los tejidos ingleses y españoles según su calidad, 1860

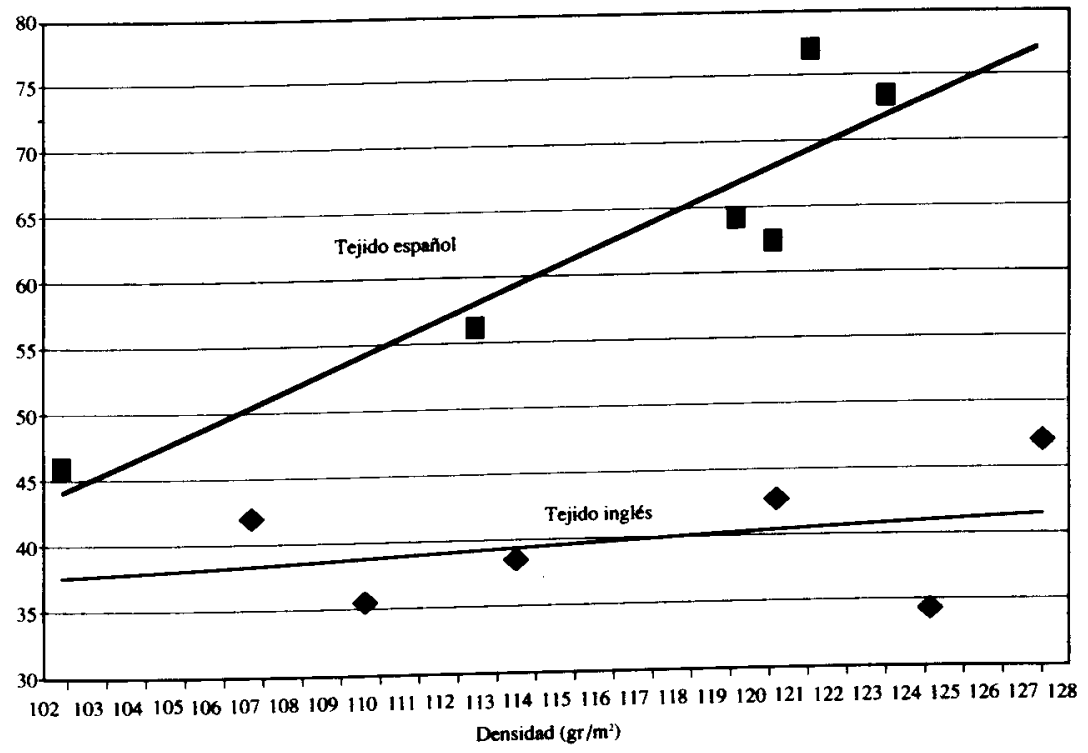

FUENTES Y NOTAS: Los precios utilizados aparecen en el Cuadro 2. Las líneas de tendencia aparecen sólo con carácter informativo puesto que los precios británicos y españoles no convergen en ningún punto. A mayor densidad, la calidad de los tejidos de algodón crece.

atención especial de los operarios y un cierto grado de humedad ambiental $^{48}$. En Lancashire, los trabajadores con más experiencia trabajaban en las fábricas de hilados finos mientras que los más jóvenes lo hacían en las fábricas de hilado más grueso. La habilidad se adquiría después de largos años de aprendizaje en las fábricas ${ }^{49}$. Por tanto, el número de operarios que se debe emplear por huso no es independiente de la calidad que se fabrica y del entrenamiento previo de la fuerza de trabajo. La comparación entre Cataluña y Gran Bretaña del número de trabajadores masculinos equivalentes (TME) por 1.000 husos equivalentes es, en este sentido, muy reveladora: pese a producir tejidos más finos, los británicos emplean 13,58 TME mientras que los catalanes 37,02 TME. En otras pala-

4k Huberman (1996).

49 Boot (1995). 
bras, los catalanes emplean más operarios para hacer funcionar la misma maquinaria que los británicos. Las empresas algodoneras españolas siempre empleaban un número excesivo de trabajadores por huso porque trataban de producir muchos productos diferentes y de una calidad superior a las capacidades de su fuerza de trabajo.

¿Cómo hubieran podido mejorar su eficiencia relativa los españoles con respecto a los británicos? Simplemente imitando a los italianos y produciendo, tal como hacían ellos, tejidos más bastos, o sea, de inferior calidad. De esta manera se podía emplear la fuerza hidráulica en vez de la del vapor, se podían emplear menos trabajadores por huso y se podía competir en aquel segmento del mercado donde los británicos no tenían ventaja comparativa ${ }^{50}$.

\section{CONCLUSIONES}

A lo largo de este artículo hemos analizado las causas de la falta de competitividad de la industria algodonera española en el mercado internacional. En este sentido, nuestros cálculos demuestran que no se puede achacar completamente a las desventajas naturales de España (el mayor coste de las materias primas) esta falta de competitividad, ya que las fábricas algodoneras españolas, pese a emplear la misma tecnología que sus homólogas británicas, eran mucho menos eficientes. La razón de esta falta de eficiencia radica en la misma protección de que gozaban los industriales algodoneros catalanes. Los altos niveles de protección condujeron a que la industria algodonera no produjese de acuerdo a su ventaja comparativa y tratase de producir bienes demasiado sofisticados para las habilidades de su fuerza de trabajo. Los industriales trataban de producir estos bienes sofisticados porque con ellos obtenían beneficios monopolísticos al tener reservado el mercado interior. Resultado de esta desviación de la ventaja comparativa fue que la industria producía de manera ineficiente, en términos comparativos, empleando demasiados trabajadores por huso. Una industria sin protección se habría especializado en un segmento diferente del mercado, menos sofisticado, en el que España e Italia disponían de mercado nacional e internacional porque eran competitivas y donde, sin duda, existían economías de escala y posibilidades de crecimiento.

${ }^{50}$ Hasta los años 1860 , las ruedas hidráulicas no se utilizaban para propulsar maquinaria produciendo tejidos finos (Von Tunzelmann, 1978). 
Nos encontramos, pues, en el caso extremo de la protección, tal como ha sido planteado por Paul Romer ${ }^{51}$. En el caso clásico, el principal efecto de la protección es la transferencia de recursos desde los consumidores hacia los productores protegidos y una pequeña pérdida de bienestar consecuencia de la mala asignación de los recursos productivos. Esta pérdida irrecuperable de eficiencia depende de la elasticidad de la oferta y de la demanda; ya que a mayor elasticidad mayor es la pérdida de eficiencia. El caso extremo, tal como ha sido planteado por Romer, sería aquel en que se produce una pérdida irrecuperable de eficiencia porque los recursos son destinados a una industria sin rendimientos crecientes en el comercio internacional, cuando podrían situarse en una industria que sí dispone de ellos. Por ejemplo, cuando se produce un bien que tiene demanda interior pero que no es posible exportar porque se hace de manera ineficiente. Aquí radica la paradoja de la industria algodonera, que perdió toda su competitividad internacional, debido, precisamente, a los aranceles.

\section{BIBLIOGRAFÍA}

A'HeArn, B. (1998): «Institutions, Externalities, and Economic Growth in Southern Italy: Evidence from the Cotton Textile Industry, 1861-1914», Economic History Review, 51, 4, pp. 734-762.

ALLEN, R. C. (1991): «Entrepreneurship, Total Factor Productivity, and Economic Efficiency: Landes, Solow, and Farrell Thirty Years Later», en P. HigONNET, D. S. LANDES y H. Rosovsky (eds.), Favorites of Fortune. Technology. Growth and Economic Development since the Industrial Revolution, Harvard (MA): Harvard University Press, pp. 203-220.

BardinI, C. (1997): «Without Coal in the Age of Steam: A Factor-Endowment Explanation of the Italian Industrial Lag before World War I», Joumal of Economic History, 57, 3, pp. 633-653.

Blaug, M. (1961): «The Productivity of Capital in the Lancashire Cotton Industry during the Nineteenth Century», Economic History Review, 13, 3, pp. 358-381.

Boot, H. M. (1995): «How Skilled Were Lancashire Cotton Factory Workers in 1833?», Economic History Review, 58, 2, pp. 283-303.

CARRERAS, A. (1983): «El aprovechamiento de la energía hidráulica en Cataluña, 1840-1920. Un ensayo de interpretación», Revista de Historia Económica, 1, 2, pp. 31-63.

CLARK, G. (1987): «Why Isn't the Whole World Developed? Lessons from the Cotton Mills», Journal of Economic History, 47, 1, pp. 141-173.

Flam, H., y Flanders, M. (1991): Heckscher-Oblin Trade Theory, Cambridge (MA), MIT Press.

51 Romer (1994). 
Gatrell, V. A. C. (1977): «Labour, Power, and the Size of Firms in Lancashire Cotton in the Second Quarter of the Nineteenth Century», Economic History Review, 30, 1, pp. 95-139.

HARLEY, C. K. (1992): «International Competitiveness of the Antebellum American Cotton Textile Industry», Joumal of Economic History, 52, 3, pp. 559-584.

Huberman, M. (1996): Escape from the Market. Negotiating Work in Lancashire, Cambridge, Cambridge University Press.

KRUGMAN, P. (1995): «Increasing Returns, Imperfect Competition and the Positive Theory of International Trade», en G. M. Grossman y K. Rogoff (eds.), Handbook of International Economics, vol. 3, Amsterdam, Elsevier, pp. 1243-1277.

Maluquer de Motes, J. (1985): «La Revolución Industrial en Cataluña», en N. Sán. CHEz-AlborNoz (ed.), La modernización económica de España, 1830-1930, Madrid, Alianza, pp. 199-225.

NADAL, J. (1974): El fracaso de la Revolución Industrial en España, Barcelona, Ariel.

- (1991): «La indústria cotonera», en J. NADAL, J. Maluquer y F. Cabana (eds.), Història Económica de la Catalunya contemporània, vol. 3, Barcelona, Enciclopèdia Catalana, pp. 12-85.

PRADOS DE LA Escosura, L. (1983): «Producción y consumo de tejidos en España, 1800-1913: Primeros resultados», en G. ANEs, L. A. Rojo y P. TEDDE (eds.), Historia económica y pensamiento social, Madrid, Alianza-Banco de España, pp. $455-471$.

- (1984): «El comercio hispano-británico en los siglos XVIII y XIX», Revista de Historia Económica, 2, 2, pp. 113-162.

Romer, P. (1994): «New Goods, Old Theory, and the Welfare Costs of Trade Restrictions», Journal of Development Economics, 42, 1, pp. 5-38.

RosÉs, J. R. (1997): «La integración vertical en el sector algodonero catalán, 1832-1861», en S. LóPEZ y J. M. VALDAliso (eds.), ¿Que inventen ellos? Tecnologia, empresa y cambio económico en la España contemporánea, Madrid, Alianza, pp. 249-280.

- (2000): «The Choice of Technology in the Mediterranean Basin: British, Spanish, Italian and US Mills Compared», en S. PAMUK y J. G. WILLIAMsON, (eds.), The Mediterranean Response to Globalization before 1850, Londres, Routhledge, pp. 134-156.

SabatE, M. (1996): El proteccionismo legitimado. Política arancelaria española a comienzos de siglo, Zaragoza, Civitas.

SÁNCHEZ, A. (1989): «La era de la manufactura algodonera en Barcelona, 1736-1839», Estudios de Historia Social, 48-49, pp. 65-113.

SANDBERG, L. (1968): «Movements in the Quality of British Cotton Textile Exports, 1815-1913», Joumal of Economic History, 28, 1, pp. 1-27.

SerRANo SANZ, J. M. (1987): El viraje proteccionista de la Restauración. La politica comercial española, 1875-1895, Madrid, Siglo XXI.

SoKolOFf, K. L. (1984): «Was the Transition from the Artisanal Shop to the Nonmechanized Factory Associated with Gains in Efficiency?: Evidence from the U. S. Manufacturing Censuses of 1820 and 1850», Explorations in Economic History, 21, 4, pp. 351-382.

SUDRIÀ, C. (1983): «La exportación en el desarrollo de la industria algodonera española, 1875-1920», Revista de Historia Económica, 1, 2, pp. 369-386. 
- (1987): «El dilema energètic en el creixement econòmic català», Revista Econòmica de Catalunya, 4, pp. 88-100.

SutTon, J. (1998): Technology and Market Structure: Theory and History, Cambridge (MA), MIT Press.

TENA, A. (1999): «Un nuevo perfil del proteccionismo español durante la Restauración, 1875-1930», Revista de Historia Económica, 17, 3, pp. 579-621.

TEnA, A., y TIRADO, D. (1996): «Protección arancelaria en la Restauración. Un debate Antonio Tena-Daniel Tirado», Revista de Economía Aplicada, 4, 11, pp. $135-150$.

Tiromson, J. (1994): Els origens de la industrialització a Catalunya. El cotó a Barcelona 1728-1832, Barcelona, Edicions 62.

Tortella, G. (1994): El desarrollo de la España contemporánea. Historia económica de los siglos XIX y $X X$, Madrid, Alianza.

Von Tunzelmann, G. N. (1978): Steam Power and British Industrialization to 1860 , Oxford, Clarendon Press.

ZamaGni, V. (1993): The Economic History of Italy, Oxford, Oxford University Press.

\section{FUENTES}

CERDÁ, I. [1968 (1867)]: Monografia estadística de la clase obrera de Barcelona en 1856, Madrid, Instituto de Estudios Fiscales.

COMISIÓN ESPECIAL ARANCELARIA (1867): Información sobre el derecho diferencial de bandera y sobre los de aduanas exigibles a los bierros, al carbón de piedra y los algodones, presentada al gobierno de Su Majestad por la comisión nombrada de efecto en Real Decreto de 10 de noviembre de 1865, Madrid, Imprenta Nacional.

Diario de Barcelona (1866): Almanaque del Diario de Barcelona para 1867, Barcelona, I. de A. Brusi.

ECONOMIST, THE (1845-1860): «Markets of the Industrial Districts». The Economist. Eluison, T. [1968 (1886)]: The Cotton Trade of Great Britain, London, Frank Cass.

FERRER VIDAL, J. (1875): Conferencias sobre el arte de bilar y tejer en general y especialmente sobre el de bilar y tejer el algodón, Barcelona, Establecimiento de Jaime Jepús Roviralta.

Giménez GuItED, F. (1862): Guia fabril e industrial de España. Publicada con el apoyo y autorización del gobierno de S. M., Madrid y Barcelona, Libreria Española y Librería del Plus Ultra.

Junta de Fábricas (1850): Censo de fábricas de 1850, Arxiu del Foment de Treball Nacional.

MADOZ, P. (1846): Diccionario geográfico-estadístico-bistórico de España y sus posesiones de Ultramar, Madrid.

Mann, J. A. [1968 (1860)]: The Cotton Trade of Great Britain, London, Frank Cass.

RonQuILlo, J. O. (1851-1857): Diccionario de materia mercantil, industrial y agrícola, Barcelona, Imprenta Gaspar.

SAYRó, E. (1842): Industria algodonera de Cataluña, Madrid, Imprenta Nacional. 